\title{
Physical Properties of DLAs: Metallicity and Neutral Hydrogen Column Density
}

\author{
J.L. Hou \\ Shanghai Astronomical Observatory, CAS, Shanghai, China \\ C.G. Shu ${ }^{1,2}$, W.P. $\mathrm{Chen}^{2}$, R.X. Chang ${ }^{1}$, C.Q. $\mathrm{Fu}^{1}$ \\ ${ }^{1}$ Shanghai Astronomical Observatory, CAS, Shanghai, China \\ ${ }^{2}$ Graduate Institute of Astronomy, National Central University, Taiwan, \\ China
}

\begin{abstract}
We investigate some basic properties of Damped Lyman alpha systems based on the Semi-Analytical model of disk galaxy formation theory. We derive the DLA metallicity, column density, number density, gas content and cosmic star formation rate by assuming that disks form at the center of dark halos, and the modelled DLAs are selected by Monte Carlo simulation according to the distributions of halo properties. We find that DLA hosts are dominated by small galaxies and biased to extended galaxies. In terms of model results, DLAs could naturally arise in a $\Lambda \mathrm{CDM}$ universe from radiatively cooled gas in dark matter halos. However, model predicts a reverse correlation between metallicity and the column density when compared with observations, regardless of the proposed observational bias. We argue that this could be resulted from the model limitations, or the inadequacy of Schmidt-type star formation mode at high redshift, or/and the diversities of DLA populations.
\end{abstract}

\section{Introduction}

Damped Lyman- $\alpha$ systems (DLAs) are absorbers seen in quasar optical spectra with HI column density $N_{\mathrm{HI}} \geq 10^{20.3} \mathrm{~cm}^{-2}$. DLAs are believed to arise in luminous galaxies or their progenitors at high redshift. But substantial debate continues over exactly what populations of galaxies are responsible for them. Current results of searches appear to suggest that galaxies giving rise to high HI column density absorbers span a wide range of morphology types, from dwarf, irregular, and low surface brightness (LSB) to normal spirals (eg. Rao et al. 2003). It is also suggested that DLAs could be associated with Lyman Break Galaxies (LBGs)(Shu 2000; Schaye 2001).

In theory, one way for DLAs research is to assume that DLAs are galaxies with different morphological types, then the model predictions can be compared with observed properties (eg. Hou, Boissier, \& Prantzos 2001; Calura, Matteucci, \& Vladilo 2003; Boissier, Péroux et al. 2003; Lanfranchi \& Friaca 2003). Another approach is to start from the framework of cosmic structure formation 
and evolution. Hence the observed DLA properties are strong tests for various cosmological models and also for Semi-Analytical Models (SAM) of galaxy formation and evolution (eg. Nagamine, Springel, \& Hernquist 2003; Cora et al. 2003).

Here, we will adopt a SAM method to examine the observed metallicity and HI column density properties of DLAs. As an illustration, DLA properties are assumed at redshift $z=3$ and the standard $\Lambda \mathrm{CDM}$ cosmogony is adopted.

\section{The model}

\subsection{Galaxy formation}

The galaxy formation model comes from Mo, Mao, \& White (1998, hereafter MMW). Detailed descriptions are given in Hou et al. (2003).

In the model of MMW, the halo mass function at any redshift $z$ can be described by the PS formalism. The distribution function of halo spin parameter can be described by a log-normal function. Disks are assumed to have exponential surface profiles $\Sigma(R)=\Sigma_{0} \exp \left(-R / R_{d}\right)$, where $\Sigma_{0}$ and $R_{d}$ are the central surface density and the scale length. The disk global properties can be uniquely determined by parameters $m_{d}, \lambda, V_{\mathrm{c}}$, and the adopted cosmogony, where $m_{d}$ is the mass ratio of disk to halo, $\lambda$ is the halo dimensionless spin parameter, $V_{\mathrm{c}}$ is the halo circular velocity.

After knowing the distributions of $V_{\mathrm{c}}$ and $\lambda$ for halos, we are able to generate a sample of disk galaxies by a Monte-Carlo simulation in the $V_{\mathrm{c}^{-}} \lambda$ plane at redshift $z \sim 3$, which is the parent sample for our follow-up DLA simulation.

\subsection{Star formation and chemical evolution}

The adopted star formation prescription comes from disk galaxy modelling of Boissier \& Prantzos (2000), in which $S F R \propto \Sigma_{g}^{1.5} V_{\text {rot }} R^{-1}$, where $\Sigma_{g}(R)$ is the gas surface density and $V_{\text {rot }}(R)$ is the rotation speed at disk radius $R$, which is calculated by considering both components of halo and disk.

The chemical evolution in disks can be expressed by the simple closedbox model with metallicity $Z$ to be $Z-Z_{i}=-p \ln \mu$, where $Z_{i}$ is the initial metallicity of gas and is assumed to be $0.01 Z_{\odot}, p$ is the effective yield, and $\mu$ is the gas fraction. We assume that at the initial time $(t=0, z=3)$, the gas surface density $\Sigma_{g 0}(R)=\Sigma_{0} \exp \left(-R / R_{d}\right)$. Star formation proceeds within disks in a typical time scale $\sim 1$ Gyr (Lanfranchi \& Friaca 2003). The effective yield $p$ is assumed to be constant and is obtained by comparing the metallicity distributions between model predictions and observations for DLAs.

\subsection{Modelled DLA population}

We investigate star formation and chemical evolution for individual disks according to the prescriptions mentioned above. The modelled DLAs are selected over the sampled galaxies by random sightlines penetrating disks according to the observed selection criterion. Random inclinations for disks in the sky are considered and a hydrogen fraction $x=0.7$ is assumed. We adopt $V_{\mathrm{c}}$ from 50 to $360 \mathrm{~km} \mathrm{~s}^{-1}$, which corresponds roughly to the observed range for spirals and irregular galaxies at the present day. 


\section{Model prediction vs observations}

\subsection{Observations}

DLAs metallicities are taken from recent compilation presented by Kulkarni \& Fall (2002), Péroux et al. (2002a) and Prochaska et al. (2003). The observed HI column densities come from the survey of Storrie-Lombardi \& Wolfe (2000) (hereafter SW00). The observed number densities and gas content of DLAs come from Péroux et al. (2002a). Low redshift gas content comes from Rao $\&$ Turnshek (2000). The observed contribution to the cosmic SFR by DLAs comes from the most recent work done by Wolfe, Gawiser, \& Prochaska(2003) (hereafter WGP03).

\section{2. $\quad V_{\mathrm{c}}$ and $\lambda$}
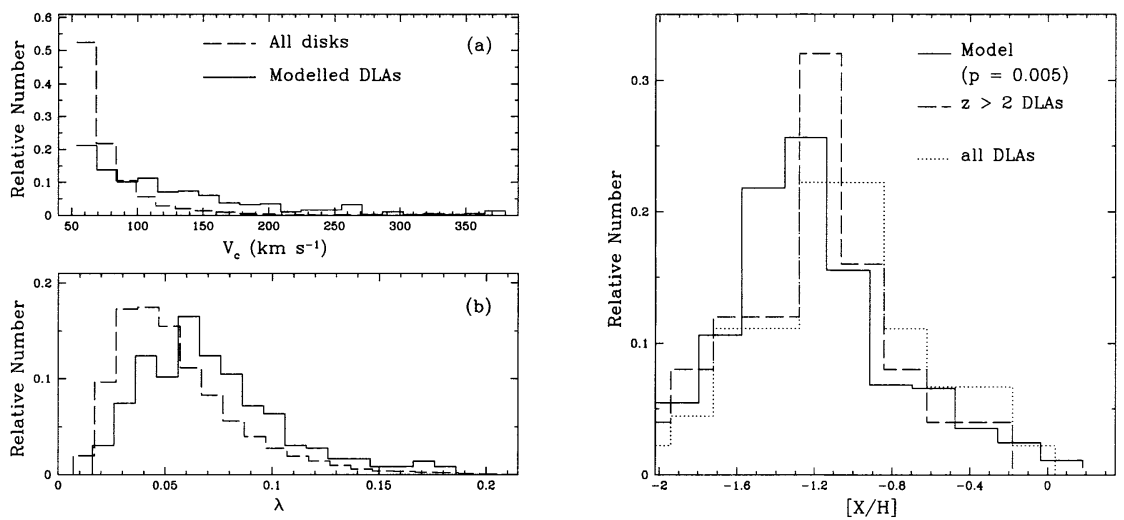

Figure 1. Left: the distributions of $V_{\mathrm{c}}$ and $\lambda$. Right: the metallicity distributions of DLAs.

In the left of Fig. 1, we present the predicted distributions of $\left(V_{\mathrm{c}}, \lambda\right)$ for the modelled DLA population. We also plot the corresponding distributions of all galaxies predicted by PS theory (dashed line). It can be found as expected that modelled DLAs are dominated by small galaxies with $V_{\mathrm{c}}<100 \mathrm{~km} \mathrm{~s}^{-1}$. The $\lambda$ distribution of selected DLAs peaks around 0.065 with the median value of 0.08 , larger than those for all galaxies. DLA hosts are extended disk galaxies.

\subsection{Metallicity distribution and effective yield}

The predicted and observed metallicity distributions of DLAs are also plotted in Fig. 1 (right). It should be noted that the predicted distribution is obtained by adjusting the effective yield $p$. We get $p=0.25 Z_{\odot}$ for the best-fit between model and observed $[\mathrm{Zn} / \mathrm{H}]$ metallicity of $z>2$ DLAs.

The obtained effective yield $p=0.25 Z_{\odot}$ is low, compared with that for the solar neighborhood (about $0.7 Z_{\odot}$ ). This is an indication of galactic winds and mass outflows during the formation of disk from dark halo (Shu, Mo, \& Mao 2003). 


\subsection{Column density, number density and gas content}
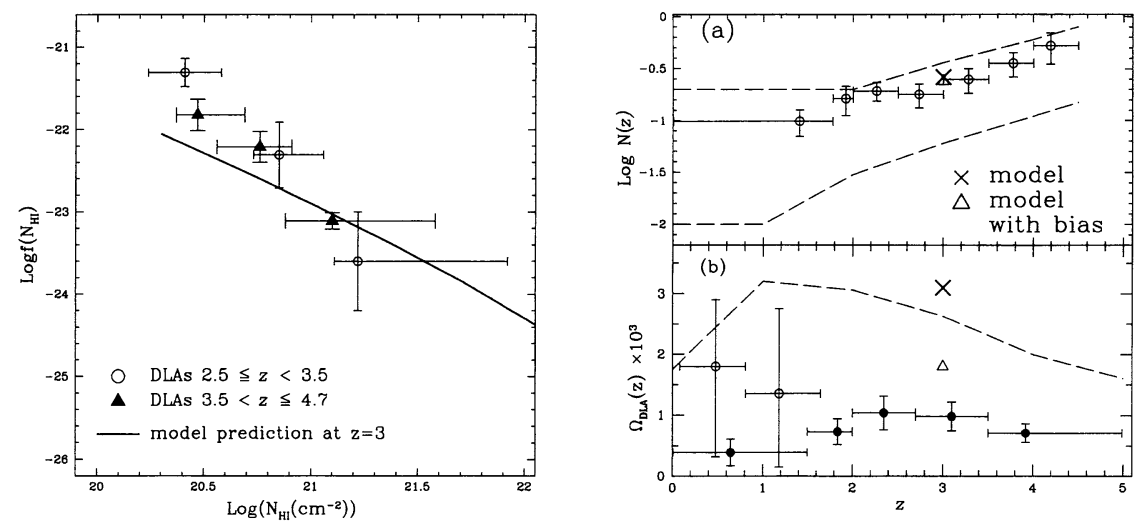

Figure 2. Left: $f\left(N_{\mathrm{HI}}\right)$ vs $N_{\mathrm{HI}}$ for DLAs. Right: (a) $N(z)$ vs $z$. The two dashed lines denote the lower and upper limits from numerical simulations (Nagamine et al. 2003); (b) $\Omega_{\text {DLA }}$ vs $z$. The dashed line is the numerical results from Cen et al. (2002).

In Fig.2, we plot the model predicted frequency distribution of column density $f\left(N_{H I}\right)$ (left, solid line), number density $N(z)$ and associated gas content $\Omega_{\text {DLA }}$ (right, cross) at $z=3$. It can be found from the figure that the predicted $f\left(N_{H I}\right)$ distribution agrees well with the high $N_{\mathrm{HI}}$ points, but not with the low

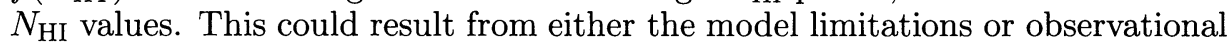
bias, or both. For instance, galactic winds and mass outflows could be important for DLAs as implied by the relative low effective yield obtained. So the closedbox model could be too relaxed.

In the lower panel of Fig. 2 (right), the triangle is the predicted DLA gas content with the consideration of the limitation suggested by Boissé et al. (1998). In general, we find that our model can well reproduce the observed DLA number density at $z \sim 3$, although the predicted neutral gas density associated with DLAs is a bit higher than that observed because the upper limit of HI column density $N_{u}$ is larger in our modelled DLAs.

\subsection{Cosmic SFR density and metallicity vs column density}

Recently WGP03 have presented the SFR density contributed by DLAs based on the CII absorption lines, which is shown in the left panel of Fig. 3 as two triangles. Our model prediction at $z=3$ is plotted as a cross. It can be found that the model prediction is consistent with observations and supports the "consensus" model described by WGP03.

A significant property of DLAs is that there seems to be an anti-correlation between observed $[\mathrm{Zn} / \mathrm{H}]$ and $\mathrm{HI}$ column density. However, as given in the right of Fig. 3, model predictions show an opposite trend compared with observations.

Several mechanisms could lead to the observed anti-correlation. First, some DLA lines of sight could penetrate galactic central regions where HI column 

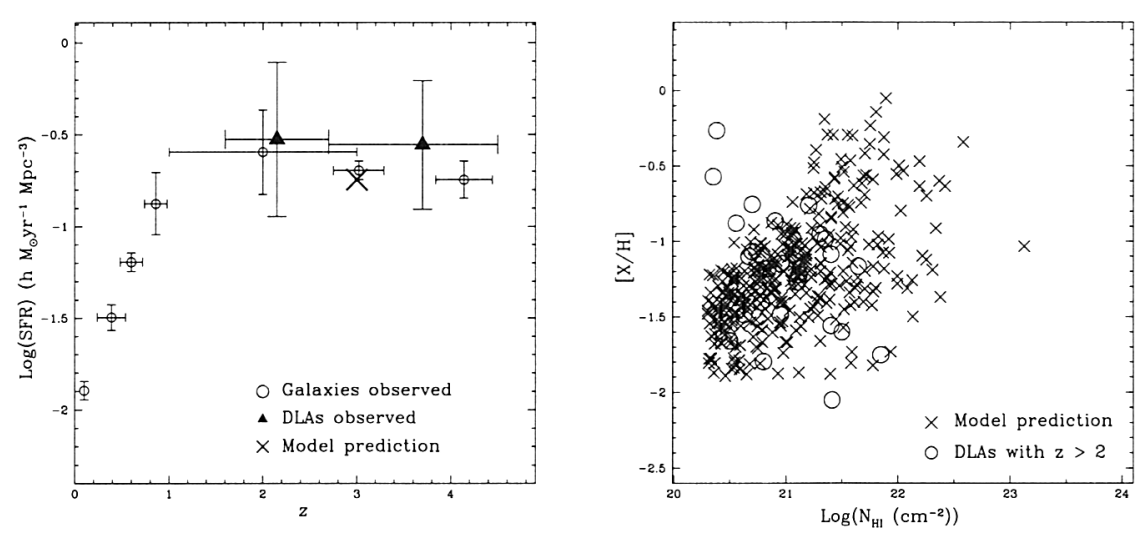

Figure 3. Right: cosmic SFR density as a function $z$. Left: The correlation between metallicity and HI column density for DLAs.

densities are low and metallicities are high. Observations of some spiral galaxies have approved this (Broeils \& van Woerden 1994). However, the absorption cross sections, hence the probability, are low for this mechanism.

Another mechanism could be the inadequacy of the adopted Schmidt type star formation prescription. Even for nearby galaxies, the physical basis of star formation is still poorly known (Kennicutt 1998; Rownd \& Young 1999). Moreover, recent observations of star formation regions in nearby galaxies done by Wong \& Blitz (2002) showed a complex relationship between SFR and $\Sigma_{\mathrm{HI}}$, and appears inconsistent with a Schmidt type law.

It is also possible that some observed points resulted from different populations of DLA galaxies. Observations suggested that DLAs could be hosted by either disks, spheroids or moving clouds within galactic halos (Maller et al. 2003). Star formation and chemical enrichment are quite different for different kinds of hosts, which could lead to the observed trend in Fig. 3.

\section{Summary}

We have generated a population of disk galaxies by SAM in the framework standard $\Lambda \mathrm{CDM}$ hierarchical picture of structure formation. Modelled DLAs are selected according to their observational criterion with the random inclination being considered.

With the effective yield $p=0.25 Z_{\odot}$ obtained for the corresponding best-fit result, our model can well reproduce the observed metallicity distribution of DLAs.

In terms of predicted results of the HI frequency distribution, the number density, gas content and cosmic SFR density at redshift 3, our model suggests that DLAs could naturally arise in a $\Lambda$ CDM universe from radiatively cooled gas in dark matter halos. 
The model predicts a positive correlation between metallicity and HI column density for DLAs, inconsistent with the observed trend. We suggest that the observed anti-correlation could most probably be physical.

Acknowledgments. We are grateful to B. Ménard, S. Boissier, W.P. Lin, H.J. Mo, D.H. Zhao for useful discussions on this subject. This work is supported by NSFC19873014, 10073016, 10173017, 10133020, NKBRSF 19990754 and NSC91-2112-M008-036. CS acknowledges the financial support of NSC for a visit to NCU and the kind hospitality during the stay in NCU.

\section{References}

Boissé, P., Le Brun, V., Bergeron, J. \& Deharveng, J.M. 1998, A\&A, 333, 841 Boissier, S., Péroux, C. \& Pettini, M. 2003, MNRAS, 338, 131

Boissier, S. \& Prantzos, N. 2000, MNRAS, 312, 398

Broeils, A.H. \& van Woerden, H. 1994, AApSS, 107, 129

Calura, F., Matteucci, F. \& Vladilo, G. 2003, MNRAS, 340, 59

Cen, R., Ostriker, J.P., Prochaska, J.X. \& Wolfe, A.M. 2003, ApJ, 598, 741

Cora, S.A., Tissera, P.B., Lambas, D.G. \& Mosconi, M.B. 2003, MNRAS, 343, 959

Hou, J.L., Boissier, S. \& Prantzos, N. 2001, A\&A, 370, 23

Hou, J.L., Shu, C.G., Chen, W.P., Chang, R.X. \& Fu, C.Q. 2003, submitted

Kennicutt, R. 1998, ApJ, 498, 541

Kulkarni, V.P. \& Fall, S.M. 2002, ApJ, 580, 732

Lanfranchi, G.A. \& Friaca, A.C.S. 2003, MNRAS, 343, 481

Maller A.H., Prochaska J.X., Somerville R.S. \& Primack J.R. 2003, MNRAS, submitted (astro-ph/0211231)

Mo, H.J., Mao, S.D.\& White, S.D.M. 1998, MNRAS, 295, 319 (MMW)

Nagamine, K., Springel, V. \& Hernquist, L. 2003, astro-ph/0302187

Péroux, C., Petitjean, P., Aracil, B. \& Srianand, R. 2002a, NewA., 7, 577

Péroux, C., McMahon, R., Storrie-Lombardi, L. \& Irwin, J. 2003, MNRAS, 346, 1103

Prochaska, J.X., Gawiser, E., Wolfe, A.M., Cooke, J. \& Gelino, D. 2003, astro$\mathrm{ph} / 0305312$

Rao, S.M., Nestor, D.B., Turnshek, D.A., Lane, W.M., Monier, E.M. \& Bergeron, J. 2003, ApJ, 595, 94

Rao, S.M. \& Turnshek, D.A. 2000, ApJS, 130, 1

Rownd, B.K. \& Young, J.S. 1999, AJ, 118, 670

Schaye, J. 2001, ApJ, 559, L1

Shu, C.G. 2000, A\&A, 354, 815

Shu, C.G., Mo, H.J. \& Mao, S.D. 2003, astro-ph/0301035

Storrie-Lombardi, L.J. \& Wolfe, A.M. 2000, ApJ, 534, 552 (SW00)

Wolfe, A.M., Gawsier, E. \& Prochaska, J.X. 2003, ApJ, 593, 235 (WGP03)

Wong, T. \& Blitz, L. 2002, ApJ, 569, 157 